\title{
VAC_16 - Duration of immunity in volunteers ten years after a dose-response yellow fever vaccine study
}

Tatiana Guimarães de Noronha ${ }^{1 *}$; Daniele Fernandes de Aguiar ${ }^{1}$; Thalita da Matta de Castro'; Ricardo Cristiano Brum ${ }^{1}$; Waleska Dias Schwarcz ${ }^{1}$; Maria de Lourdes de Sousa Maia' ${ }^{1}$; Akira Homma ${ }^{1}$.

${ }^{1}$ Fiocruz/Bio-Manguinhos.

Introduction: The problem of inadequate supply of yellow fever vaccine (YFV) is recurrent, due to a combination of limited production capacity and expansion of circulation of the yellow fever virus. In 2009 Bio-Manguinhos did a dose-response study with the YFV, administrated in the usual mean dose of 27,476 IU and in decreasing doses (10,447 IU, 3013 IU, 587 IU, 158 IU and 31 IU), by the usual subcutaneous route and usual volume $(0.5 \mathrm{~mL})$. The decreasing doses were obtained by dilution in the laboratory of the manufacturer and the lots in test had industrial quality. Around 30 days after the vaccination, doses down to 587 IU had similar immunogenicity to the full dose. Seropositivity was maintained for 10 months in these doses. Eight years after that dose-response study, at least $80 \%$ of the subjects who had seroconverted after yellow fever vaccination with doses from 27,476 IU down to 31 IU. Seropositivity and antibody titers in the reduced-dose groups were comparable to that of the full dose. To complete this issue, a 10 years-duration of immunity study was done.

Objective: To evaluate the duration of immunity 10 years after the dose-response study of 2009, by measuring the level of neutralizing antibodies.

Methodology: Phase IV cohort study, in young healthy adults who received YFV during the dose-response study in 2009, were seronegative before vaccination and not revaccinated. Serology for neutralization against yellow fever was done in all participants. The criteria for seropositivity to yellow fever will be evaluated according to the reciprocal calculation of the dilution and its correlate with the standard in $\mathrm{mIU} / \mathrm{mL}$ : seropositive (antibody titers equal to or greater than 1: 100 or $3.15 \mathrm{mUI} / \mathrm{mL}$ ); undetermined serum (antibody titers equal to or greater than 1:71 and antibody titers equal to or less than 1:99); and seronegative (titers equal or less than 1:70). Comparison of each group rate of seroprotection and antibody neutralizing levels to the reference group was done.

Results: A total of 253 participants adhered to the study protocol. Groups of decreasing doses have rates of seropositivity to yellow fever ranging from $83.8 \%$ to $93.0 \%, 10$ years later. The difference of rates of seropositivity by group are statistically non-significant ( $p$-value 0.700 ) and there is no significance in the difference of seropositivity between each reduced dose and standard dose. For geometric mean titers, all groups are similar (p-value 0.896) and there is no statistically significance in the GMT ratio for each reduced dose with standard dose.

Conclusion: Considering that protection can be inferred from those immunological parameters, the current study supports the use of yellow fever vaccine in reduced doses, and particularly the fractionation of vaccine, to face sudden increased demand and insufficient supply of vaccine.

Keywords: yellow fever vaccine immunogenicity; dose-response study; duration of immunity 